\title{
Démographie et environnement à Madagascar
}

Demography and environment in Madagascar

\section{Bénédicte Gastineau et Frédéric Sandron}

\section{(2) OpenEdition}

\section{Journals}

Édition électronique

URL : http://journals.openedition.org/economierurale/921

DOI : 10.4000/economierurale.921

ISSN : 2105-2581

Éditeur

Société Française d'Économie Rurale (SFER)

Édition imprimée

Date de publication : 1 novembre 2006

Pagination : 41-56

ISSN : 0013-0559

Référence électronique

Bénédicte Gastineau et Frédéric Sandron, « Démographie et environnement à Madagascar 》, Économie rurale [En ligne], 294-295 | Juillet-octobre 2006, mis en ligne le 23 octobre 2009, consulté le 30 avril 2019. URL : http://journals.openedition.org/economierurale/921 ; DOI : 10.4000/economierurale.921 


\section{Démographie et environnement à Madagascar}

Bénédicte GASTINEAU • Institut de recherche pour le développement, UMR 151 LPED Université de Provence-IRD

Frédéric SANDRON • Institut de recherche pour le développement, UMR 151 LPED Université de Provence-IRD

\section{Introduction}

D ans les études sur le développement durable, la démographie fait figure de parent pauvre. La variable démographique, à Madagascar comme ailleurs, est souvent présentée comme étant à l'origine de la pauvreté et de la dégradation de l'environnement. Elle est alors considérée comme une variable exogène au système. Pourtant, la relation population-environnement dans le contexte malgache actuel est digne d'être étudiée de manière précise et détaillée, et ce pour trois raisons. Primo, les sociétés humaines et les systèmes écologiques sont très diversifiés au sein de cette grande île, réputée à raison pour son endémisme et sa biodiversité. Les lois récentes de décentralisation et de régionalisation, l'action locale aussi bien des administrations publiques que des ONG incitent à une connaissance fine et "sur le terrain » des processus. Ceci permettrait une synergie plus grande entre les résultats de la recherche et leur utilisation directe dans l'élaboration de politiques d'aménagement ou de développement durable. Secundo, les recherches et les politiques sont encore très sectorielles et peu tournées vers une approche interdisciplinaire ou complexe. Tertio, la croissance démographique la plus forte de la population malgache est à venir. La transition de la fécondité est tout juste commencée, puisqu'après la baisse de la mortalité entamée vers 1950, la fécondité n'a baissé sensiblement qu'à partir de la fin des années 1990. Le contexte démographique doit donc être intégré plus que jamais dans les années à venir dans les politiques de développement durable.

Pour étudier la place de la composante démographique dans la question du développement durable, il est proposé ici dans une première partie de faire un cadrage général méthodologique sur la considération scientifique et idéologique de la variable démographique dans ses rapports à l'environnement. Nous verrons notamment le glissement de paradigme opéré à la fin des années 1980. À partir de cette grille de lecture, il s'agira dans une deuxième partie d'étudier la place de la variable démographique dans les recherches et dans les politiques menées à Madagascar en matière environnementale. Nous verrons notamment que des recherches récentes sur la thématique population-environnement plaident pour une prise en compte de la complexité de cette relation, s'inscrivant ainsi dans la mouvance scientifique étudiée dans la première partie. Résultats à l'appui, la troisième partie sera consacrée à la présentation d'une opération de recherche effectuée en milieu rural malgache, en guise d'illustration de ce paradigme de recherche. La conclusion donnera alors quelques recommandations méthodologiques pour appréhender le processus du développement durable dans toute sa complexité.

\section{Population et environnement : quels paradigmes ?}

Dans l'étude de la relation population-environnement, trois courants sont aisément repérables : l'approche malthusienne ou néo-malthusienne, l'approche boserupienne et une approche plus récente, plus nuancée, que l'on qualifiera de « complexe ». 


\section{La croissance démographique, fléau pour l'environnement}

Lorsqu'elles devinrent un champ d'étude à part entière dans les années 1960, les relations population-environnement s'inscrivaient dans une grille d'analyse fortement imprégnée des idées malthusiennes. Le postulat de départ était clair : la croissance démographique a des effets néfastes sur l'environnement. C'était le message délivré en 1968 par l'ouvrage d'Ehrlich «The Population Bomb » ou par le célèbre rapport Meadows publié en 1972 sous le titre «The Limits to Growth ». Analysant les relations entre la démographie, la nourriture, la pollution, l'industrialisation et les ressources non renouvelables sur la base d'un système d'équations non linéaires, la conclusion était que seule une réduction drastique de la croissance démographique permettrait d'éviter une catastrophe écologique. On retrouve l'argumentation malthusienne originelle selon laquelle si les freins préventifs (recul de l'âge au mariage, mise au monde d'enfants uniquement si l'on a les moyens de les élever) ne sont pas instaurés par les individus, ce sont les freins répressifs (famine, guerre, épidémie) qui le feront pour eux (Malthus, 1798).

D'un point de vue démographique, on peut noter que ce courant néo-malthusien est dominant à l'époque où la croissance de la population mondiale, et surtout celle des pays du tiers-monde, est maximale, c'est-àdire dans les décennies 1960 et 1970. Les travaux qui s'y rattachent ont eu des répercussions très importantes sur l'opinion publique et sur les décideurs. Ils ont en effet donné une assise scientifique à la mise en place de nombreux programmes de planification familiale. Malgré une vive opposition des pays en développement ${ }^{1}$ à ces programmes lors de la première Conférence de population à Bucarest en 1974, nombre d'entre eux comme l'Algérie, le

1. Slogan phare de l'époque parmi ces pays : « $L a$ meilleure pilule, c'est le développement $»$.
Mexique ou la Chine mettront en place très rapidement des politiques antinatalistes.

\section{La croissance démographique, mère de l'innovation}

Pour s'opposer aux théories néo-malthusiennes, il y eut une théorie alternative qui inversait leur postulat de départ. L'argument de Boserup, dont l'ouvrage fondateur de 1965 fournit une analyse historique des rapports entre la croissance démographique et les types de système agraire, est que la croissance démographique est un aiguillon qui va forcer l'adoption d'un nouveau système agraire plus performant pour permettre de nourrir la population. Dans la thèse boserupienne, comme dans celle de Malthus d'ailleurs, l'homme est plutôt apathique par nature et il préfère utiliser un système agraire moins intensif donc moins nécessiteux en facteur travail, à moins d'y être contraint, justement par la pression démographique. Boserup (1976) prolongera cette théorie à l'environnement selon le même raisonnement pour adopter des techniques respectueuses de son intégrité. Simon (1985) sera un ardent défenseur de cette thèse.

\section{La croissance démographique, une variable parmi d'autres}

Si les théories néo-malthusiennes et boserupiennes peuvent paraître concurrentes, elles ont en tout cas le point commun de ne pas être nuancées dans leurs conclusions. Picouet et al. (2004) les rangent dans la catégorie des théories « déterministes ». Il faut cependant remarquer que leur portée d'origine est extrêmement large, que ce soit dans le temps ou dans l'espace, et que le véritable problème, d'un point de vue scientifique, est celui de leur transposition à des échelles plus fines. Car, à partir de la fin des années 1980, lorsque les chercheurs se sont penchés de manière plus précise sur des études de cas, il s'est avéré que les relations entre la démographie et l'environnement ne pouvaient pas se contenter d'être appréhendées en simples termes malthusiens ou boserupiens. Le concept de 
« développement durable » proposé en 1987 par le rapport Bruntland et vulgarisé par le Sommet de la Terre de Rio en 1992 intégrait ce changement de paradigme.

Issu de ces réflexions, le chapitre $\mathrm{v}$ de l'Agenda 21 intitulé «Dynamique démographique et durabilité », reflète assez bien les préoccupations d'une nouvelle communauté scientifique dont les recherches se sont multipliées depuis les années $1990^{2}$. Plus diffus que les courants néo-malthusiens ou boserupiens, ces travaux, que nous regrouperons sous la bannière du «paradigme de la complexité », intègrent quelques principes dont on peut lister les principaux :

- premièrement, ils refusent d'accabler la seule croissance démographique comme facteur de dégradation de l'environnement ; - deuxièmement, pour expliquer l'impact anthropique sur l'environnement, ils prennent en compte une série de variables autre que la croissance démographique, comme par exemple la technologie, les modèles de consommation, les politiques publiques, la culture, la répartition des terres et des richesses, ou encore les modes d'organisation ;

- troisièmement, ils affinent les échelles, les questions de recherche, les concepts; - quatrièmement, ils essaient de réfléchir sur une méthodologie de l'interdisciplinarité, indispensable pour aborder la thématique population-environnement.

Après ce bref rappel sur les courants scientifiques faisant le lien entre démographie et environnement, voyons comment cette relation a été étudiée à Madagascar.

2. Cf. par exemple : Commoner (1991); Domenach et Picouet (2000) ; Le Bras (1994) ; Mathieu (1998) ; Pebley (1998) ; Tabah (1995) ; Tabutin et Thiltgès (1992) ; Tiffen (1993).

\section{Population et ressources à Madagascar : un équilibre malthusien ?}

Dans l'histoire de l'Afrique précoloniale, les écrits prêtent souvent aux populations des pratiques agricoles traditionnelles et une sorte de sagesse qui leur permettaient de gérer harmonieusement leur environnement et de conserver un certain équilibre population-ressources. La population dans ce schéma est contrôlée selon un schéma malthusien. La colonisation est alors présentée dans ce système comme une double rupture de l'équilibre ancien. D'une part, elle désorganise les systèmes de production agricole traditionnels, d'autre part, en introduisant la médecine moderne et en limitant les fléaux, elle va à l'encontre des « freins naturels » de la croissance de la population. À Madagascar, cette thèse est défendue notamment par Chevalier $(1952)^{3}$ : «Si l'équilibre ancien entre les ressources et les populations risque de se trouver compromis d'ici quelques décades, par l'intervention puissante des techniques occidentales appliquées à la lutte contre la mortalité, du moins les progrès récents effectués par ces mêmes techniques, dans d'autres domaines de la recherche et de l'action, permettent-ils d'envisager, grâce à une meilleure connaissance des ressources et de leur utilisation, l'établissement progressif d'un nouvel équilibre ».

\section{L'époque précoloniale et le mythe de l'équilibre ancien}

Il y a une forte incertitude sur le nombre d'habitants pendant le XIX ${ }^{\mathrm{e}}$ siècle avant le protectorat et la colonisation française. Campbell (1991) tente à partir de sources d'origines diverses de reconstituer l'évolution de la population entre 1818 et 1900 . En 1818, la population avoisinerait les 2 millions et en 1900, il n'y aurait guère plus de 2,5 millions d'individus sur l'île.

3. Voir en particulier la page 171 . 
Cependant, l'effectif de population varie beaucoup au gré des crises de subsistances ou des épidémies.

On peut faire plusieurs lectures de l'évolution démographique de Madagascar au $\mathrm{XIX}^{\mathrm{e}}$ siècle. La première interprétation est malthusienne : la population est fortement limitée par les ressources et par l'environnement. Compte tenu des techniques de production agricole rudimentaires, les densités de population auraient atteint leurs « limites naturelles » à la fin du XVIII ${ }^{\mathrm{e}}$ siècle aboutissant à un effectif maximal de population de 2 à 3 millions d'habitants (Campbell, op . cit.). Au cours du $\mathrm{XIX}^{\mathrm{e}}$ siècle, la population aurait progressé jusqu'en 1867, mais sans réel progrès en matière de technique de production agricole, elle aurait dépassé ses « limites naturelles » et les freins répressifs malthusiens seraient entrés en action : épidémies de variole, famines et paludisme.

Campbell propose une autre lecture de l'équilibre entre la population et les ressources au XIX ${ }^{\mathrm{e}}$ siècle, durant la période précoloniale. Pour l'auteur, dès la fin du $\mathrm{XVIII}^{\mathrm{e}}$ siècle ce sont les facteurs politiques et non pas naturels qui sont au centre de la relation entre la population et les ressources. Les choix politiques de l'État Merina ${ }^{4}$ ont eu un impact sur la croissance démographique. Dans un premier temps, il est positif grâce à une meilleure organisation de la production agricole. Dans un second temps, l'intensification du fanompoana $a^{5}$ afin de mobiliser de la main-d'œuvre pour la construction d'infrastructures, pour la mise en valeur de terres et le renforcement de l'armée, a ralenti la dynamique démographique en désorganisant l'économie agricole et en séparant pour de longues périodes les époux, faisant donc diminuer la fécondité. Ces conditions de vie imposées par le fanompoana ont rendu les populations particulièrement vulnérables aux maladies.

4. Ethnie des Hautes Terres malgaches, de la région de l'Imerina.

5. Corvée obligatoire par servitude royale.
Du fait des corvées de servitude, de nombreuses terres sont restées inexploitées et recouvertes d'eau stagnante, ce qui a favorisé la multiplication des moustiques, et notamment des anophèles responsables des épidémies de paludisme. La crise de mortalité des années 1870 a donc aussi une explication politique.

Les autorités administratives de l'île au $\mathrm{XIX}^{\mathrm{e}}$ siècle avaient déjà légiféré sur les ressources naturelles. Dès le début du XIX ${ }^{\mathrm{e}}$, les forêts appartenaient à l'État. La royauté merina qui unifia le pays en 1829 interdisait le déboisement pour des raisons, semble-t-il, de sécurité intérieure. Le $t a v y^{6}$ devint alors un moyen d'expression contestataire des populations vis-à-vis du pouvoir royal (Henkels, 1999). En 1868, avec le Code des 101 Articles et en 1881 avec le Code des 305 Articles, le brûlis et l'installation de nouveaux habitants en forêt furent interdits. Cette politique avait surtout pour objectif de contrôler des ressources naturelles jugées d'une grande valeur (Henkels, op. cit.).

Pour Campbell, pendant la période précoloniale à Madagascar, la dynamique démographique et sa relation avec les ressources naturelles ne répondent donc finalement pas à une logique malthusienne mais à une logique politique dont le résultat final est une croissance démographique quasiment nulle au cours du XIX ${ }^{\mathrm{e}}$ siècle. À la fin de ce siècle, le nombre des hommes est faible, à tel point que ce " sous-peuplement » semble être un obstacle à la colonisation de Madagascar. Certains missionnaires envoyés sur la Grande Ile pour faire un inventaire écologique et humain mettent en garde le gouvernement sur le fait qu'il sera très difficile de mettre en valeur et d'exploiter les richesses d'un si grand territoire avec une main-d'œuvre aussi peu nombreuse (Grandidier, 1872).

6. Culture sur brûlis. 
Figure 1. Évolution de la population à Madagascar de 1921 à 2050

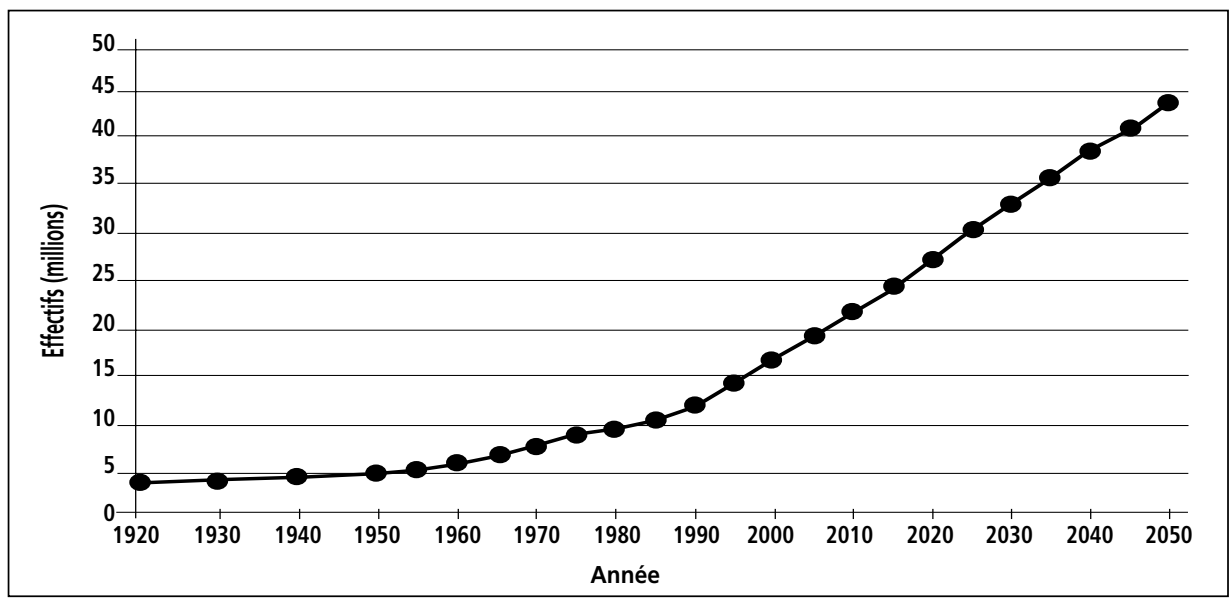

Sources : Commissariat général au Plan, 1962, pour la période 1921-1949 United Nations, 2005, pour la période 1950-2050 (scénario central pour les projections)

2. Le protectorat (1885-1895) et la colonisation française (1896-1960) : stagnation démographique et déforestation

L'administration coloniale voit donc le faible effectif de population comme une contrainte: "Quand, en effet, il y a une population si disséminée dans un pays d'une telle étendue, on ne saurait y rencontrer une main-d'œuvre considérable » écrit Piolet (1898) ${ }^{7}$. La mise en valeur de terres agricoles, l'exploitation des ressources minières et les grands travaux publics se heurtent à la difficulté de recruter des travailleurs. En 1896, les autorités coloniales abolissent l'esclavage à Madagascar, ce qui permet de rendre disponible une main-d'œuvre jusqu'alors servile.

L'effectif de la population augmente très faiblement jusqu'en 1940 (figure 1). La densité est faible puisqu'en 1911, on compte alors 4,8 habitants au kilomètre carré. Sur un tiers du territoire, la densité est inférieure à 2 habitants au kilomètre carré et elle ne dépasse les 20 habitants que sur une très faible part de l'île (environ $5 \%$ de l'espace total) (De Martonne, 1911). En 1936, la densité serait passée à 6,4 habitants au kilo-

7. Cf. page 258 . mètre carré (Andriamanana, 1939), comptant toujours de très fortes disparités avec une succession d'espaces vides et de terres plus densément peuplées (Chevalier, 1952). Il faut attendre 1946 pour que la croissance démographique s'accélère grâce à la baisse de la mortalité, rendue possible par les succès enregistrés contre la peste et le paludisme (Chevalier, op. cit.).

Pendant le protectorat et la colonisation, le problème de population à Madagascar est donc avant tout perçu comme un problème de sous-peuplement. Les faibles densités n'empêchent cependant pas que l'exploitation des ressources naturelles de l'île soit particulièrement importante. Selon Jarosz (1993), la forêt primaire aurait ainsi largement régressé entre 1895 et 1925 . L'État colonial inventorie, préserve les ressources, particulièrement les forêts, de l'exploitation traditionnelle de la part des populations malgaches, notamment pour les exploiter à des fins commerciales (Sauvaire, 1903). Ce que redoute le plus l'administration, c'est l'agriculture itinérante et plus particulièrement le tavy. Selon Chevalier $(1922)^{8}$, « en premier, il faut attribuer

8. $C f$. page 474 . 
le déboisement au nomadisme des populations forestières qui déplacent sans cesse leurs cultures et souvent leurs villages pour les établir constamment sur l'emplacement de la forêt abattue ». L'administration va donc tenter de contrôler les cultures sur brûlis et instaurer des campagnes de reboisement obligatoires.

Dès 1927, un décret permet la création de dix réserves naturelles affranchies de tous droits d'usage. Toute forme d'exploitation (chasse, pêche, récolte de produits naturels, etc.) y est interdite et il est prévu d'encourager la migration des jeunes adultes et l'expulsion des individus qui commettraient des délits tels que le tavy (Molet, 1957). À propos des zones frontalières d'une réserve, Molet (1955) écrit : «Les rives de la Lokoho et de l'Androranga seront bientôt surpeuplées, c'est-à-dire que le rapport entre les terres cultivables et les populations qu'elles peuvent nourrir, en l'état actuel des techniques, deviendra inquiétant. Pour éviter que les éléments jeunes ne se taillent des champs aux dépens de la forêt, il conviendrait de les dériver vers d'autres régions ».

En 1935, un Code forestier institue une réglementation répressive de l'usage des forêts à Madagascar. Dans le même temps, l'intensification de l'agriculture, la culture de rente (girofle, café, vanille, poivre), la réalisation de grands périmètres rizicoles, le commerce de bois etc., ont largement contribué au déboisement et à l'appauvrissement des sols. En outre, comme au $\mathrm{XIX}^{\mathrm{e}}$ siècle, le tavy reste un mode de protestation des paysans face au pouvoir en place (Kull, 2002).

Cet épisode de déforestation à Madagascar durant la colonisation est donc observé à un moment où la croissance de la population est faible et où le tavy est interdit (Jarosz, 1993). Jusqu'au début des années 1950, la croissance démographique n'est pas considérée comme un problème pour

9. $C f$. page 7 . l'environnement. Ce n'est donc pas l'effectif de la population qui fait peur mais ses pratiques agricoles et principalement celle du tavy. Par conséquent, la conservation de l'environnement est souvent imposée à des populations qui se trouvent dépossédées des ressources naturelles.

\section{Après 1960 : à la recherche d'un nouvel équilibre?}

Après l'Indépendance, la croissance démographique s'accélère. Le taux de croissance annuel de la population passe audessus du seuil des $2 \%$ au début des années 1960. En 1966, une enquête démographique indique un effectif de population de 6,2 millions. La fécondité reste forte et la baisse de la mortalité observée depuis 1950 perdure, ce qui explique que le rythme de croissance de la population s'accélère. Au recensement de 1975, l'île compte 7,6 millions d'habitants. En 1993, ils sont plus de 12 millions et plus de 18 millions en 2005 (Razafimanjato et al, 2001 ; United Nations, 2005). Le taux d'accroissement annuel atteint alors $3 \%$. Notons qu'au début du $\mathrm{XXI}^{\mathrm{e}}$ siècle, les trois quarts des Malgaches vivent en milieu rural. Pour les années à venir, la population atteindrait 32,3 millions d'habitants en 2030 et 43,5 millions en 2050 selon l'hypothèse centrale des projections des Nations Unies (2005).

De l'Indépendance en 1960 au début des années 1980, ni la croissance démographique ni la dégradation des ressources naturelles n'apparaissent comme une question politique à Madagascar. D'une part, la politique de population sous la Première République (1958-1972) est plutôt nataliste et dans la décennie 1970 de la Seconde République, la question de la croissance démographique est complètement occultée (Raison-Jourde, 1993). D'autre part, la déforestation ainsi que la pratique du brûlis sont activement encouragées entre 1975 et 1985 (Banque mondiale, 2003a). La Constitution de 1960 confirme le droit de propriété privée 
sur la terre mais à condition que l'exploitation individuelle se fasse dans l'intérêt collectif. À ce titre, elle autorise l'État à confisquer une terre de plus de cinq hectares qui resterait inexploitée. Ceci a évidemment incité à une exploitation « effrénée » des terres.

L'idée d'un lien causal entre la croissance de la population, l'agriculture itinérante et la déforestation apparaît à Madagascar au cours des années 1980 (Jarosz, op. cit.). La croissance démographique est alors présentée par certains comme une des premières causes de la dégradation de l'environnement (Huffy et al, 1998 $)^{10}$. Cette idée est affirmée aussi bien dans les politiques d'environnement que dans les politiques de population. Dans la Politique nationale de population pour le développement économique et social de 1990 (loi $\mathrm{n}^{\circ}$ 90-030), l'environnement occupe une place importante et le texte ne laisse aucun doute sur l'effet négatif de la croissance démographique sur les ressources naturelles : «L'homme est au centre de la dégradation de l'environnement en tant qu'auteur et victime. La poussée démographique des dernières décennies a accentué, d'une part la pression sur les terres, et d'autre part la dévastation des forêts pour satisfaire les besoins de survie de la population, alors que le développement durable repose sur l'utilisation rationnelle des ressources pouvant répondre aux besoins actuels de la population et assurer l'avenir des générations futures $\gg{ }^{11}$. Dans les faits, les stratégies d'actions $^{12}$ étaient trop vastes, trop vagues et le

10. Huffy M., Chollet M., Razakamanantosa A. Le programme d'action environnemental à Madagascar : conservation et néocolonialisme. Documents de travail EPB, décembre $1998, \mathrm{n}^{\circ} 1$.

11. $C f$. page 26 .

12. «Prendre des mesures conséquentes quant à la disparition progressive des forêts, de la dégradation des prairies et de la baisse de la fertilité des sols ; Promouvoir l'utilisation à grande échelle de l'énergie alternative produite localement; Prendre des mesures visant à protéger la Zone Économique Exclusive Maritime, notamment contre les déversements des déchets toxiques... » (p. 26). ministère de la Population n'a pas disposé des moyens nécessaires face à cet ambitieux programme. En outre, cette politique « a souffert de l'instabilité politique marquant les années $1990 »\left(\right.$ INSTAT et ORC Macro 2005) ${ }^{13}$.

Du côté des institutions chargées de la préservation de l'environnement, le ton est souvent le même : "La vitesse de déforestation est liée à la croissance démographique. La problématique de l'environnement se présente de manière aiguë en termes de déséquilibres entre les ressources naturelles d'une part (terres cultivables, eaux, végétations ligneuses, etc.), et d'autre part les besoins accrus de la population en croissance rapide à la recherche d'une amélioration générale de leur condition de vie» $(\mathrm{ONE}, 2002)^{14}$. Mais là encore, les actions sont restées très sectorielles. Peu d'opérations transversales ont été mises en place, même si des cellules de réflexion sur la question environnementale ont été installées dans certains ministères, comme celui en charge de la Population.

Face à ce discours « malthusien » qui se situe donc à un niveau macro, des études de terrain montrent que le lien entre population et environnement est loin d'être univoque et que la démographie n'explique pas à elle seule l'état de dégradation ou de préservation d'un environnement.

\section{Quelques études de cas}

Les quelques résultats issus des études citées ci-dessous menées à Madagascar indiquent au contraire une diversité des interactions entre les variables démographiques et environnementales. On peut situer ces travaux dans un courant de recherche qui, sans pour autant négliger la variable démographique, n'en fait pas pour autant un bouc émissaire des problèmes d'environnement. Pour reprendre l'expression de Picouet et al. (2004) $)^{15}$, ce courant ne

13. $C f$. en particulier la page 4 .

14. $C f$. page 113 .

15. Voir les pages 18-19. 
résume pas les relations entre population et environnement à la « simple relation surpopulation-pauvreté-dégradation ».

Ainsi, revisitant en 1992 un terroir étudié en 1966 par Bonnemaison, BlancPamard et al. (1997) constatent de nombreux changements en matière environnementale et agricole. L'espace de la commune de Tsarahonenana, jugé quasiment saturé en 1966 au regard du système de production, a pu néanmoins accueillir un nombre croissant d'habitants grâce à des dynamiques interne et externe qui ont permis des innovations en matière agricole et environnementale : aménagement des pentes par processus anti-érosifs, reboisement de la partie montagneuse par des pins, meilleure maîtrise des conditions hydrologiques, association agriculture/élevage, intensification des cultures. Dans une autre région des Hauts Plateaux, Locatelli (2000) montre bien la diversité des stratégies des individus indépendamment de la densité démographique. L'enclavement, la pauvreté, l'instabilité politique, les représentations sociales de la nature sont des variables déterminantes dans la relation entre la population et l'environnement. Dans le même esprit, Keck et al. (1994) stipulent dans une étude sur une zone rurale autour du Parc national d'Andasibe que les principales causes du tavy sont la pauvreté, la très faible productivité de l'agriculture et l'absence d'infrastructures. La croissance de la population est un des facteurs identifiés mais elle n'est pas présentée comme une cause principale.

S'intéressant à un autre type d'écosystème que les précédents, Case et al. (2004) identifient dans la région du Sud-Ouest de Madagascar un grand nombre de causes à la déforestation autres que la croissance démographique telle que l'insuffisance et la fluctuation des revenus agricoles, l'absence de droits fonciers, le développement de la culture du maïs encouragée par des prix à l'achat élevés. Dans une commune de cette région, dans la forêt des Mikea, Blanc-Pamard et al. (2005) précisent que cette logique commerciale est surtout le fait de quelques gros producteurs puisque, pour une population de 20000 habitants, une vingtaine d'exploitants sont responsables de la moitié des défrichements annuels. Dans cette même région, les taux très élevés de déforestation s'expliquent aussi par l'immigration des populations Antandroy qui, par besoin d'argent et notamment pour acheter du bétail, dégradent la forêt qui était auparavant protégée par un ensemble de croyances et de tabous respectés par la population locale (Banque mondiale, 2003b). Plus que l'aspect démographique de la migration, c'est ici son aspect socioéconomique qui prévaut.

L'ensemble de ces études et l'analyse historique proposée montrent qu'une vision déterministe et mécaniste de l'impact de la population sur l'environnement à Madagascar est erronée. Les systèmes population-ressources sont complexes, divers et plus que la densité démographique ou l'effectif de la population, de nombreux facteurs politiques, économiques, culturels ou sociaux influencent la dégradation ou la préservation de l'environnement.

\section{Population et environnement : prendre en compte la complexité}

Pour illustrer ces propos, nous allons préciser quelques mécanismes mettant en relation les variables démographiques, économiques et environnementales dans une zone rurale des Hautes Terres malgaches. 


\section{Aspects méthodologiques}

Les résultats qui vont être présentés sont ceux d'un programme de recherche intitulé « Dynamique démographique et développement durable $(4 D) »^{16}$. Il étudie les liens entre les dynamiques démographiques, économiques et l'utilisation du milieu naturel. La zone d'étude se situe dans les Hautes Terres malgaches. Plus précisément, il s'agit de neuf fokontany ${ }^{17}$ de la commune d'Ampitatafika, sur la RN7 à mi-chemin d'Antsirabe et de la capitale Antananarivo, peuplés de 1621 ménages. La riziculture y est l'activité dominante.

Dès la conception du programme, le choix a été fait de se porter sur une approche fine des relations population-environnement, au risque de ne pas être représentatif pour d'autres régions. En revanche, la méthode reste transposable. Le protocole de recherche reste assez simple mais a été monté de manière à ce que les recommandations des différents textes et recherches passés issus de ce que nous avons appelé le " paradigme de la complexité » soient rendues effectives et opérationnelles. Les questions de l'interdisciplinarité, du traitement des échelles et de l'espace (SIG, analyse spatiale et modélisation), des apports réciproques du quantitatif et du qualitatif, du recours à des cadres théoriques population-environnement ont été au centre de la réflexion du montage du programme $^{18}$.

L'hypothèse de travail retenue est qu'en situation de pauvreté, le risque et l'incertitude sont moteurs dans les comportements

16. Les partenaires de recherche sont : Institut de recherche pour le développement (UMR 151 LPED), École supérieure des sciences agronomiques d'Antananarivo, Institut catholique de Madagascar, Institut national de la statistique de Madagascar, Laboratoire FORUM de l'Université Paris-X, Laboratoire POPINTER de l'Université Paris-V, ministère de la Population de Madagascar, PACT Madagascar.

17. Equivalent de « village » ou « hameau ».

18. Pour plus de détails : http://www.ird.fr/ madagascar/4d des familles, dans tous les domaines de la vie. La réduction de cette incertitude passe donc par des stratégies démographiques et d'utilisation des ressources naturelles qui viennent renforcer une montée en puissance des stratégies économiques de pluriactivité. La majorité des relations entre variables démographiques et variables d'utilisation du milieu naturel sont médiatisées par les variables économiques, incluant celles ayant trait au système de production agricole. C'est d'ailleurs à notre sens une des faiblesses des approches étudiant les relations entre la croissance démographique et la qualité de l'environnement que de ne pas inclure ces aspects économiques à l'interface des deux sphères.

\section{Relations population-environnement à Ampitatafika}

À Ampitatafika, la quasi-totalité des ménages ont une activité agricole. La riziculture domine et la plupart des agriculteurs pratiquent d'autres types de culture comme le manioc, le maïs, les haricots verts, etc. Les techniques de production sont traditionnelles : le labour se fait le plus souvent avec l'angady (bêche traditionnelle) et peu d'engrais chimiques sont utilisés. Les techniques modernes de production sont souvent trop coûteuses et ne sont pas toujours adaptées. Le labour attelé est par exemple une technique difficile à mettre en œuvre sur des parcelles de surface très réduite. Les familles sont très dépendantes des ressources naturelles dont la quantité et la qualité déterminent leurs conditions de vie et leur revenu.

L'idée que l'état de l'environnement dans la commune s'est dégradé est largement partagée par les ménages enquêtés par le programme $4 D$. Les deux tiers des chefs de ménage constatent qu'au cours des vingt années passées, les surfaces boisées ont régressé. Beaucoup (88\%) souhaitent d'ailleurs que le reboisement s'intensifie dans la commune d'Ampitatafika. Les corvées de bois pour assurer les besoins quotidiens sont de plus en plus coûteuses en 
temps et nécessitent une plus grande disponibilité de la main-d'œuvre. De même, selon $40 \%$ des chefs de ménage, la fertilité des sols aurait diminué depuis dix ans, chiffre à mettre en parallèle avec les $43 \%$ de chefs de ménage qui constatent une baisse de leur rendement en riz.

La majorité des individus perçoivent donc leur environnement comme une contrainte à leur pratique agricole et comme une variable majeure quant à leurs conditions de vie. Dans ce contexte, deux questions méritent d'être posées :

- quelles sont les caractéristiques des ménages ou individus qui par leur activité agricole ont des pratiques plus aptes à garantir une meilleure qualité et fertilité des sols ? - quelles sont aujourd'hui les capacités et les stratégies d'adaptations des individus et des ménages compte tenu du fait qu'ils perçoivent leur environnement comme dégradé ?

\section{Données}

Les données utilisées sont d'abord celles de l'Enquête référence du programme $4 D$. Cette enquête a été réalisée en 2003 auprès de 1621 ménages, soit la totalité des résidents de 9 fokontany. L'enquête a porté sur les conditions de vie, les caractéristiques économiques, démographiques des individus et des ménages mais aussi sur leurs pratiques agricoles ainsi que sur leur perception de l'environnement et de la pauvreté. Ensuite, nous utiliserons des entretiens réalisés à Ampitatafika ${ }^{19}$ permettant d'illustrer les résultats quantitatifs.

Sur les 1621 chefs de ménage de la zone d'étude, $97 \%$ d'entre eux soit 1578 exploitent des terres. Parmi eux, $99 \%$ exploitent des parcelles en tanety (colline) et $96 \%$ des parcelles en plaine. Le nombre moyen de parcelles est de dix. La possession de parcelles dans les deux systèmes de culture est

19. Quarante entretiens réalisés par Tahiry Rabeandriamaro et Juliette Rafanjaniriana à partir d'une grille élaborée par Frédéric Gannon et Frédéric Sandron. donc généralisée, elle répond à la fois à un mode de transmission des terres par héritage et à une nécessité économique de lissage du risque inhérent à l'activité agricole. Les six premiers indicateurs sont liés au système de culture vivrier au sein duquel les associations culturales sont rares mais où la rotation culturale est forte. Quant au dernier indicateur, il concerne les parcelles d'eucalyptus, dont un intérêt est de limiter l'érosion.

Nous allons d'abord tenter à partir de ces données de déterminer quelles sont les variables qui distinguent les ménages en matière de gestion des sols. Compte tenu des données disponibles, nous avons choisi de construire un indice permettant de diviser les ménages en trois groupes distincts de taille comparable. Cet indice, qui varie de 0 à 7 , tient compte des informations suivantes : - le ménage pratique ou ne pratique pas la jachère ;

- le ménage pratique ou ne pratique pas la rotation des cultures ;

- le ménage fait ou ne fait pas de compostage ;

- le ménage utilise ou n'utilise pas de fumier pour fertiliser le sol de ses parcelles ;

- le ménage a protégé ou n'a pas protégé ses terres contre l'érosion par des haies vives ; - le ménage a protégé ou n'a pas protégé ses terres contre l'érosion par des diguettes; - le ménage a planté ou n'a pas planté d'arbres sur ses parcelles l'année précédant l'enquête.

Chaque ménage s'est vu attribuer un indice compris entre 0 et 7 . Trois groupes ont été constitués : du groupe qui a le plus de pratiques favorables au maintien de la fertilité des sols (groupe 3) à celui qui en a le moins (groupe 1). Les valeurs seuil retenues pour la délimitation des groupes l'ont été de manière à constituer trois ensembles d'effectif comparable. Ensuite, nous avons cherché les relations entre ces groupes et un grand nombre de variables économiques (revenu, type d'activité principale, etc.), démographiques (âge du chef de ménage, 
nombre d'enfants, etc.), agricoles (superficie exploitée, type de cultures, etc.). Les résultats présentés ici mettent en exergue les variables qui expliquent le mieux les différences entre les groupes : la formation et l'information en matière d'agriculture, l'alphabétisation, la superficie des terres exploitées, le revenu et le nombre d'individus résidents dans le ménage.

\section{Résultats}

Dans la zone d'étude, la très large majorité des paysans se sentent concernés par les problèmes d'environnement : $95 \%$ des chefs de ménage déclarent d'ailleurs que l'ensemble des individus sont concernés par ceux-ci. Ils ont tout à fait conscience du lien entre les activités humaines et la dégradation ou la protection de l'environnement. Par conséquent, ils savent aussi qu'ils peuvent changer leurs pratiques pour protéger ou améliorer la qualité de leurs sols.

«Nos terres sont un peu épuisées, il nous faut beaucoup d'engrais, chimique et biologique. Même le compost, nous voulons apprendre à le faire ici. Moi je connais les méthodes mais je n'ai pas les moyens de les appliquer. Ici, nous ne pouvons pas mettre en jachère nos terres, il faut les cultiver tous les ans. Donc, le mieux c'est de faire la rotation de culture » (agriculteur, Ampitatafika, 2004).

" J'aimerais apprendre une technique qui me permette de reconnaître la qualité $d u$ sol, pour pouvoir l'améliorer selon nos besoins, car voyez-vous, on y consacre toutes nos forces en cherchant tous les moyens pour y parvenir mais il nous manque cette connaissance qui peut vraiment nous aider à améliorer notre activité » (agriculteur, Ampitatafika, 2004).

Le faible degré de connaissances techniques limite en effet fortement l'innovation dans les pratiques agricoles. Rares sont les agriculteurs d'Ampitatafika qui déclarent avoir reçu une formation ou une aide technique en matière agricole : moins de $10 \%$ ont reçu l'année précédant l'enquête la visite d'un technicien et seuls $16 \%$ ont pu suivre une formation agricole depuis le début de leur activité. Gannon et Sandron (2006) montrent qu'à Ampitatafika, les agriculteurs peuvent faire preuve d'innovation si le risque d'échec d'une nouvelle pratique est quasiment nul. L'aide et la connaissance techniques aident beaucoup à limiter ce risque, ainsi que les mécanismes d'imitation des paysans entre eux.

«On a vu les voisins qui ont pratiqué, et on les a copiés. C'est toujours comme ça à la campagne, si les gens ont vu quelqu'un faire quelque chose de nouveau, on vient lui demander comment on fait et on essaie après » (agriculteur, Ampitatafika, 2004).

«À partir du moment où les gens constatent les avantages d'une nouvelle technique, alors ils seront favorables à son adoption » (agriculteur, Ampitatafika, 2004).

«Il est très difficile de faire sortir les paysans de leurs habitudes. Il faut une personne sensibilisatrice sur place qui peut les encadrer et là ils vont suivre petit à petit leurs conseils pour adopter l'innovation dans leur pratique » (agriculteur, Ampitatafika, 2004).

On constate effectivement dans l'Enquête référence $4 D$ que les agriculteurs formés ou aidés techniquement sont plus nombreux à avoir des pratiques favorables au maintien de la fertilité des sols (tableau 1). L'adoption de ces pratiques est aussi plus fréquente dans les ménages dont le chef est alphabétisé. Le fait de savoir lire et écrire est une variable favorable : l'accès à l'information est facilité ainsi que le fait d'imposer une nouvelle technique vis-à-vis de son entourage social ou familial. Les individus éduqués sont plus aptes aussi à s'affranchir de la coutume. 
Tableau 1. Répartition des ménages suivant diverses variables à Ampitatafika (en \%)

\begin{tabular}{|c|c|c|c|c|c|}
\hline & Groupe 1 & Groupe 2 & Groupe 3 & Total $(\%)$ & Effectifs \\
\hline \multicolumn{6}{|c|}{ Ont reçu une visite } \\
\hline Oui & 20,6 & 29,1 & 50,3 & 100,0 & 155 \\
\hline Non & 29,2 & 37,8 & 33,0 & 100,0 & 1423 \\
\hline \multicolumn{6}{|c|}{ Khi-deux $=18,703 * * *$} \\
\hline \multicolumn{6}{|c|}{ Ont reçu une formation agricole } \\
\hline Oui & 19,1 & 30,0 & 50,9 & 100,0 & 220 \\
\hline Non & 29,9 & 38,1 & 32,0 & 100,0 & 1358 \\
\hline \multicolumn{6}{|c|}{ Khi-deux $=30,587 * * *$} \\
\hline \multicolumn{6}{|c|}{ Sait lire et écrire } \\
\hline Oui & 26,4 & 36,0 & 37,6 & 100,0 & 1082 \\
\hline Non & 32,5 & 38,7 & 28,8 & 100,0 & 496 \\
\hline
\end{tabular}

Khi-deux $=13,521 * * *$

Superficie de terres exploitées (hectares)

\begin{tabular}{|c|c|c|c|c|c|}
\hline Moins de 0,25 & 33,3 & 38,0 & 28,6 & 100,0 & 768 \\
\hline 0,25 et plus & 23,7 & 35,9 & 40,4 & 100,0 & 810 \\
\hline
\end{tabular}

Khi-deux $=28,978 * * *$

Nombre d'individus dans le ménage

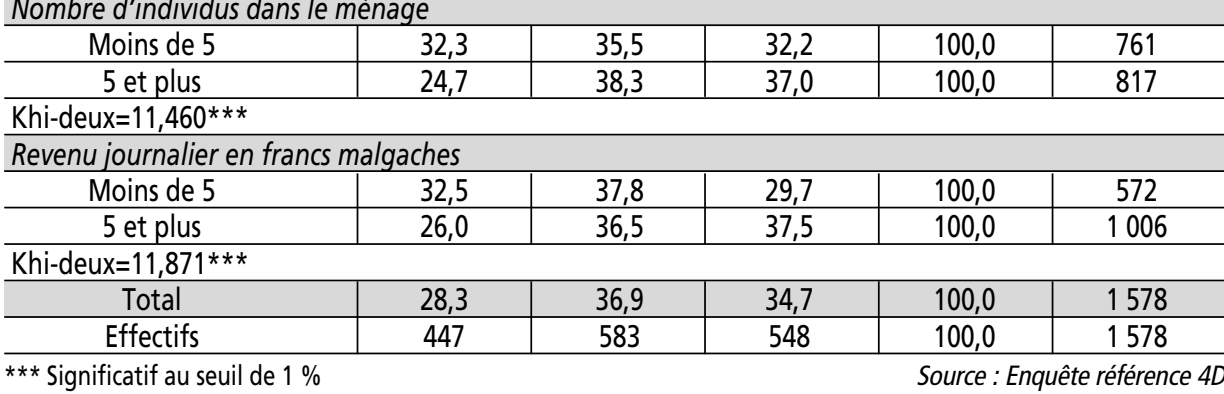

De même, plus la superficie de la terre exploitée est importante, plus les pratiques préservant la fertilité du sol sont nombreuses au sein des ménages agricoles. Certains investissements et aménagements, ceux contre l'érosion, sont plus faciles à engager sur des grandes parcelles que sur des petites parcelles ${ }^{20}$. Le revenu joue un rôle important, car la mise en place puis l'entretien des

20. Un résultat intéressant est que les pratiques des paysans sont en général similaires sur toutes les parcelles de même nature (bas-fonds vs plaine). Par exemple, pour l'innovation du repiquage en ligne par rapport au repiquage en foule, les paysans adoptent la nouvelle technique suivant une logique $\mathrm{du}$ « tout ou rien », ne testant pas sur une de leurs parcelles la nouvelle technique mais attendant suffisamment longtemps pour être certain de son rendement supérieur et l'adoptant alors sur toutes les parcelles à la fois (Gannon et Sandron, 2003). Le nombre de parcelles et un éventuel effet de lissage du risque ne jouent donc pas ici. aménagements ont un coût et les ménages les plus pauvres peuvent hésiter à prendre des risques en modifiant leurs techniques de culture. Ils cherchent avant tout à assurer leur autosubsistance.

Enfin, un résultat intéressant est que plus le nombre d'individus résidents dans le ménage est grand, plus le ménage met en œuvre des pratiques agricoles protectrices de la fertilité des sols. Cette relation s'explique par le fait notamment que certaines familles de faible effectif peuvent manquer de maind'œuvre pour certaines pratiques agricoles (construction et entretiens des diguettes et des haies vives, etc.).

Les quelques variables identifiées comme ayant une relation avec les pratiques agricoles sont assez classiques : la pauvreté, l'absence de formation agricole, le manque d'éducation ne favorisent pas l'adoption de techniques agricoles qui permettraient de 
mieux préserver la fertilité des sols. En revanche, il est intéressant de noter que ce sont les ménages qui ont le plus de membres résidents qui sont les plus aptes à le faire.

On peut se demander maintenant quelles sont les capacités et possibilités d'adaptation des familles dans ce contexte de pauvreté, de forte densité où la taille réduite des parcelles et la faible fertilité de certaines terres limitent les rendements agricoles. Quelles sont les stratégies démographiques, économiques et quels sont leurs impacts sur l'environnement?

Les stratégies les plus fréquentes consistent à multiplier les sources de revenus via des activités non agricoles ou des activités agricoles en dehors d'Ampitatafika. Environ un cinquième des actifs ont une seconde activité non agricole au moment de l'enquête en 2003. La plupart sont vendeurs de légumes, de fruits ou artisans (menuisier, briquetier, maçon). Mais ces activités sont saisonnières, elles dépendent du calendrier agricole et des besoins en argent. Une enquête menée à Ampitatafika au mois de décembre 2004 en période de soudure et d'augmentation du prix du riz a montré qu'en situation de crise, les ménages diversifiaient et multipliaient davantage leurs activités (Binet et $a l ., 2005)$. Cependant, quelle que soit l'importance des activités non agricoles, rares sont les familles qui abandonnent totalement l'agriculture. La migration et notamment la migration saisonnière vers des zones de culture comme Ambatondrazaka, ou vers la capitale Antananarivo est une autre forme de diversification possible. Certaines de ces stratégies aident à préserver l'environnement en limitant la pression sur les terres. D'autres, en revanche, sont plutôt un facteur de dégradation. Par exemple, la fabrication de briques est une pratique particulièrement nocive pour la fertilité des sols.

Cette étude locale menée à Ampitatafika a permis de spécifier quelques mécanismes d'interaction entre population et environnement. Un des résultats marquants ici est que l'effectif du ménage et donc le nombre d'enfants ne peuvent finalement pas être considérés comme le facteur principal et déterminant de la dégradation des ressources naturelles. Entre la population et l'environnement, le rôle d'interface du système de production économique et agricole est ici confirmé, comme cela a été écrit par BlancPamard et Milleville $(1985)^{21}$ : «Une réalité agraire résulte de rapports complexes entretenus entre une société et son environnement ».

\section{Conclusion}

S'il fallait synthétiser les parties précédentes de cet article, nous dirions que dans l'étude des relations population-environnement, les schémas simplistes et mécaniques, qu'ils soient d'obédience malthusienne ou boserupienne ont sans doute des vertus explicatives à des échelles de temps et d'espace très vastes mais sont en général d'un recours assez limité lorsqu'il s'agit de faire une analyse sur des territoires ou des laps de temps plus restreints que ce soit dans une optique de recherche, de recherche-action ou de développement. Le passage du micro au macro, invoquant là encore le temps et l'espace, reste alors la grande question à résoudre, mais ceci n'est pas spécifique à la relation population-environnement, elle hante en effet quasiment toutes les disciplines et les champs scientifiques.

Cette mise en garde contre ces grands schémas théoriques ne signifie pas qu'il faille dénier tout rôle actif à la variable démographique dans la dynamique de l'environnement ; ce serait, comme disait Malthus, « renverser l'arc dans l'autre sens à force de vouloir trop le recourber $»$. Il s'agit simplement de faire la part des choses et de s'intéresser à un ensemble plus vaste de variables et à leurs interactions.

21. $C f$. page 101 . 
Si l'on se place maintenant dans une optique de gestion durable de l'environnement à Madagascar, la question est alors de savoir comment passer d'une approche scientifique de type «complexe » à une élaboration de politique intégrée. Une proposition de réflexion pourrait donc être d'identifier des secteurs ou des thématiques à l'interface des questions de population et celle d'environnement, comme par exemple le secteur crucial du foncier (MAEP, 2005). Dialectiquement, ceci per- met de prendre en compte la complexité des phénomènes tout en la réduisant à certains sous-systèmes jugés pertinents. Ces recommandations en faveur d'une approche intégrée sont donc très banales mais leur mode d'opérationnalisation reste un sujet d'actualité. Chercheurs, décideurs et acteurs du développement doivent continuer de mener une réflexion en ce sens pour que les maillons de la chaîne recherche/décision/action restent attachés les uns aux autres.

\section{RÉFÉRENCES BIBLIOGRAPHIQUES}

Andriamanana F. (1939). Contribution à l'étude du problème démographique de Madagascar. Les presses modernes, $102 \mathrm{p}$.

Banque mondiale (2003a). Madagascar. Revue du secteur rural et environnemental. Volume I : rapport principal. Document de la Banque mondiale, rapport $\mathrm{n}^{\circ} 26106-\mathrm{MG}, 69 \mathrm{p}$.

Banque mondiale (2003b). Madagascar. Revue du secteur rural et environnemental. Volume II : Annexes techniques. Document de la Banque mondiale, rapport $\mathrm{n}^{\circ} 26106-\mathrm{MG}, 167 \mathrm{p}$.

Binet B., Briet P., Gastineau B., Gastineau P., Omrane M. (2005). Conditions de vie des ménages dans la commune rurale d'Ampitatafika en période de soudure. Conséquences de la hausse du prix du riz sur les pratiques agricoles et la formation du revenu (décembre 2004 - février 2005). Travaux et Documents $n^{\circ} 1$, Programme $4 D$, Institut catholique de Madagascar et Institut de recherche pour le développement, Antananarivo, 18 p.

Blanc-Pamard C., Bonnemaison J., Rakoto Ramiarantsoa H. (1997). Tsarahonenana 25 ans après. Un terroir « où il fait toujours bon vivre ». In Blanc-Pamard C., Boutrais J. (Eds.), « Thèmes et variations : nouvelles recherches rurales au Sud», Editions de l'ORSTOM, p. 25-61.

Blanc-Pamard C., Milleville P. (1985). Pratiques paysannes, perception du milieu et système agraire. In Blanc-Pamard C., Lericollais A. (Eds.), «Dynamique des systèmes agraires, à travers champs agronomes et géographes », Editions de l'ORSTOM, p. 101-138.

Blanc-Pamard C., Milleville P., Grouzis M., Lasry F., Razanaka S. (2005). Une alliance de disciplines sur une question environnementale : la déforestation en forêt des Mikea (Sud-Ouest de Madagascar). Natures Sciences Sociétés, vol.13, p. 7-20.

Boserup E. (1965). Évolution agraire et pression démographique. Traduction française, 1970, Paris, Flammarion.

Boserup E. (1976). Environment, Population and Technology in Primitive Societies. Population and Development Review, vol, $\mathrm{n}^{\circ}$ 1, p. 257-270.

Campbell G. (1991). The State and Pre-colonial Demographic History: the case of nineteenth-century Madagascar. Journal of African History, 32, p. 415-445.

Case T., Milhøj A., Ranaivoson S., Randriamanarivo J. (2004). Causes of Deforestation in Southwestern: what do we 
know? Forest policy and economics, 6 , p. 33-48.

Chevalier A. (1922). La végétation à Madagascar d'après l'ouvrage de H. Perrier de La Bathie. Annales de Géographie, vol. 31, p. 405-484.

Chevalier L. (1952). Madagascar : population et ressources. Paris, Cahiers de l'INED, $\mathrm{n}^{\circ} 15,212 \mathrm{p}$.

Commissariat général au plan (1962). Économie Malgache. Évolution $1950-$ 1960, République Malgache. Paris, Commissariat général au plan, 277 p.

Commoner B. (1991). Croissance démographique rapide et pression sur l'environnement. In Tapinos G., Blanchet D., Horlacher D.-E. (Eds.), «Conséquences de la croissance démographique rapide dans les pays en développement $»$, Paris, INED, p. 145-175.

De Martonne E. (1911). La densité de la population à Madagascar. Paris, Annales de Géographie, vol. 20, p. 77-85.

Domenach H., Picouet M. (2000). Population et environnement. Paris, PUF, Collection Que-Sais-Je ?, $\mathrm{n}^{\circ} 3556,128$ p.

Ehrlich P.-R. (1968). The Population Bomb. New York, Ballantine Books Inc., 152 p.

Gannon F., Sandron F. (2006). Échange, réciprocité et innovation dans une communauté paysanne. Une lecture conventionnaliste, Économie Rurale, $\mathrm{n}^{\circ} 292$, p. 50-67.

Grandidier A. (1872). Rapport sur une mission à Madagascar 1868-1871. Paris, Imprimerie nationale, Archives des Missions Scientifiques, tome VII, p. 445-477.

Henkels D. (1999). Une vue de près du droit de l'environnement malgache. African Sudies Quartely, vol. 3, $\mathrm{n}^{\circ}$ 2, $12 \mathrm{p}$.

INSTAT, ORC Macro (2005). Enquête Démographique et de Santé de Madagascar 2003-2004. Calverton, Maryland, USA, 442p.

Jarosz L. (1993). Defining and Explaining Tropical Deforestation: Shifting Cultivation and Population Growth in Colonial Madagascar (1896-1940). Economic
Geography, vol. 69, Issue 4, p. 366-379. Keck A., Sharma N.-P., Feder G. (1994). Population Growth, shifting cultivation, and unsustainable agricultural development: a case of study from Madagascar. World Bank Discussion paper, Africa Technical department series, $\mathrm{n}^{\circ} 234$, Washington D.C., 63 p.

Kull C. (2002). Madagascar aflame: landscape burning as peasant protest, resistance, or a resource management tool? Political Geography, 21, p. 927-953.

Le Bras H. (1994). Les limites de la planète. Mythes de la nature et de la population. Paris, Flammarion, 349 p.

Locatelli B. (2000). Pression démographique et construction du paysage rural des tropiques humides : l'exemple de Mananara (Madagascar). Doctorat en Sciences de l'Environnement, ENGREF, 430 p. MAEP (2005). Lettre de politique foncière. Direction des Domaines et des Services Fonciers, ministère de l'Agriculture, de l'Élevage et des Pêches, République de Madagascar, $11 \mathrm{p}$.

Malthus T.-R. (1798). Essai sur le principe de population en tant qu'il influe sur le progrès futur de la société, avec des remarques sur les théories de M. Godwin, de M. Condorcet et d'autres auteurs. Paris, INED, 1980, 166 p.

Mathieu P. (1998). Population, pauvreté et dégradation de l'environnement en Afrique : fatale attraction ou liaisons hasardeuses ? Natures, Sciences, Sociétés, vol. $6, \mathrm{n}^{\circ} 3$, p. 27-34.

Molet L. (1955). Notes sur les villages de la zone périphérique de la réserve naturelle $n^{\circ} 12$ dite du Marojejy - Oct.-nov. 1955. Office de la Recherche Scientifique et technique d'Outre-mer, série sociologieethnologie, Cahier n ${ }^{\circ} 2466,18$ p.

Molet L. (1957). Les problèmes humains posés par les réserves naturelles intégrales à Madagascar, Office de la Recherche Scientifique et technique d'Outre-mer, série sociologie-ethnologie, Cahier $\mathrm{n}^{\circ} 3055,21 \mathrm{p}$. 
ONE (2002). Analyse diagnostic du littoral Est (Soanierana Ivongo - Brickaville). Rapport final, Office National pour l'Environnement, Ministère de l'Environnement, Antananarivo, $128 \mathrm{p}$.

Pebley A. (1998). Demography and the Environment. Demography, vol.35, $\mathrm{n}^{\circ} 4$, p. 377-389.

Picouet M., Boissau S., Brun B., Romagny B., Rossi G., Sghaier M., Weber J. (2004). Le renouvellement des théories population-environnement. In Picouet M., Ghaier M., Genin D., Abaab A., Guillaume H., Elloumi M. (Eds), «Environnement et sociétés rurales en mutation. Approches alternatives », Paris, IRD Editions, p. 17-43.

Piolet J.-B. (1898). Douze leçons à la Sorbonne sur Madagascar. Son état actuel, ses ressources, son avenir. Paris, Augustin Challamel Editeur, 407 p.

Raison-Jourde F. (1993). Une transition achevée ou amorcée. Politique Africaine, $\mathrm{n}^{\circ} 52$, p. 6-18.

Rapport Meadows (1972). Halte à la croissance. Paris, Fayard, (trad.fr).

Razafimanjato J., Randriamanjakosoa J.-H., Rabeza V., Rakotondrajaona N., Allman
J. (2001). La situation démographique à Madagascar. Population, vol.56, $\mathrm{n}^{\circ} 4$, p. 657-668.

Sauvaire C. (1903). Les bois à Madagascar. Bulletin de la Société Commerciale de Bordeaux, $\mathrm{n}^{\circ}$ 1-2, p. 167-181.

Simon J. (1985). L'homme, notre dernière chance. Paris, PUF.

Tabah L. (1995). Les interrelations population-environnement-développement : état de la question et perspectives. In Clarke J.-I., Tabah L. (Eds.), «Population-Environment-Development-Interactions », Paris, CICRED, p. 33-65.

Tabutin D., Thiltgès E. (1992). Relations entre croissance démographique et environnement. Du doctrinal à l'empirique. Revue Tiers Monde, vol.33, n¹30, p.273294.

Tiffen M. (1993). Productivity and Environmental Conservation under Rapid Population Growth: a case of Machakos District. Journal of International Development, vol.5, $\mathrm{n}^{\circ} 2$, p. 207-223.

United Nations (2005). World Population Prospects - The 2004 Revision. Analytical Report, Department of Economic and Social Affairs, Population Division. 